Volume 70, Number 1, Pages 269-278(2021)

DOI: $10.31801 /$ cfsuasmas.771172

ISSN 1303-5991 E-ISSN 2618-6470

Received by the editors: July 18, 2020; Accepted: December 20, 2020

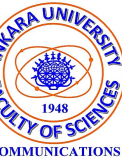

\title{
SEVERAL INTEGRAL INEQUALITIES FOR GENERALIZED RIEMANN-LIOUVILLE FRACTIONAL OPERATORS
}

\author{
Juan Gabriel GALEANO DELGADO ${ }^{1}$, Juan E. NÁPOLES VALDÉS ${ }^{2}$, \\ and Edgardo PÉREZ REYES ${ }^{1}$ \\ ${ }^{1}$ Facultad de Ciencias e Ingeniería, Universidad del Sinú Elías Bechara Zainúm, \\ Montería, COLOMBIA \\ ${ }^{2}$ UNNE, FaCENA Ave. Libertad 5450, Corrientes 3400, ARGENTINA \\ ${ }^{2}$ UTN-FRRE, French 414, Resistencia, Chaco 3500, ARGENTINA
}

\begin{abstract}
In this paper, using a generalized integral operator, of the RiemannLiouville type, defined and studied in a previous work by the authors, we obtain various integral inequalities for positive functions, which contains several reported in the literature. Various remarks carried out throughout the work and pointed out in the Conclusions, show the scope and strength of our results, in particular, it is shown that under particular cases of the considered kernel, several known fractional integral operators are obtained.
\end{abstract}

\section{INTRODUCTION}

Calculus, using different notions of derivatives and integrals of arbitrary order, has become in recent years one of the centers of attention of mathematical researchers, both pure and applied.

By other hand, one of the most developed mathematical areas in the last 20 years is that of Integral Inequalities, associated with different functional notions: convex, synchronous functions within the framework of Riemann, fractional and generalized integral operators.

Throughout the work we use the functions $\Gamma$ (see $8,9,10,11)$ and $\Gamma_{k}$ (cf. defined by 2]):

2020 Mathematics Subject Classification. Primary 26A33; Secondary 26D10, 47A63.

Keywords and phrases. Generalized fractional operator, Riemann-Liouville integral, integral inequalities.

凶juangaleanod@unisinu.edu.co; jnapoles@exa.unne.edu.ar-Corresponding author; edgardomath@gmail.com

(D) 0000-0002-3042-933X; 0000-0003-2470-1090; 0000-0002-7666-1636.

(C)2021 Ankara University Communications Faculty of Sciences University of Ankara-Series A1 Mathematics and Statistics 


$$
\begin{aligned}
& \Gamma(z)=\int_{0}^{\infty} \tau^{z-1} e^{-\tau} \mathrm{d} \tau, \quad \Re(z)>0, \\
& \Gamma_{k}(z)=\int_{0}^{\infty} \tau^{z-1} e^{-\tau^{k} / k} \mathrm{~d} \tau, k>0 .
\end{aligned}
$$

It is clear that if $k \rightarrow 1$ we have $\Gamma_{k}(z) \rightarrow \Gamma(z), \Gamma_{k}(z)=(k)^{\frac{z}{k}-1} \Gamma\left(\frac{z}{k}\right)$ and $\Gamma_{k}(z+k)=z \Gamma_{k}(z)$. As well, we define the $k$-beta function as follows

$$
B_{k}(u, v)=\frac{1}{k} \int_{0}^{1} \tau^{\frac{u}{k}-1}(1-\tau)^{\frac{v}{k}-1} d \tau
$$

notice that $B_{k}(u, v)=\frac{1}{k} B\left(\frac{u}{k}, \frac{v}{k}\right)$ and $B_{k}(u, v)=\frac{\Gamma_{k}(u) \Gamma_{k}(v)}{\Gamma_{k}(u+v)}$.

In 3 the following fractional integral operator of the Riemann-Liouville type is defined and its main properties are studied.

Definition 1. The k-generalized fractional Riemann-Liouville integral of order $\alpha$ with $\alpha \in \mathbb{R}$, and $s \neq-1$ of an integrable function $\chi(u)$ on $[0, \infty)$, are given as follows:

$$
s J_{F, a_{1}}^{\frac{\alpha}{k}} \chi(u)=\frac{1}{k \Gamma_{k}(\alpha)} \int_{a_{1}}^{u} \frac{F(\tau, s) \chi(\tau) d \tau}{[\mathbb{F}(u, \tau)]^{1-\frac{\alpha}{k}}},
$$

with $F(\tau, 0)=1$ and $\mathbb{F}(u, \tau)=\int_{\tau}^{u} F(\theta, s) d \theta$.

In this work, it was shown that many of the known integral operators can be obtained as particular cases of them.

The main purpose of this paper, using the generalized fractional integral operator of the Riemann-Liouville type, from Definition 1 1 , is to establish several integral inequalities, which contain as particular cases, several of those reported in the literature.

\section{MAIN RESULTS}

Below we present several integral inequalities, in the framework of the operators of Definition 1 the first of them is the following.

Theorem 2. Let $\varphi$ be a positive non-decreasing continuous function on $\left[a_{1}, a_{2}\right]$ and let $h:\left[a_{1}, a_{2}\right] \rightarrow \mathbb{R}^{+}$be a positive continuous function. Then for $a_{1}<\tau \leq a_{2}$, $\theta>0, \sigma>0, \alpha>0$ and $s \neq-1$, we have

$s J_{F, a_{1}}^{\frac{\alpha}{k}}\left[\left(\tau-a_{1}\right)^{\sigma} h(\tau) \varphi^{\theta}(\tau)\right]^{s} J_{F, a_{1}}^{\frac{\alpha}{k}} h(\tau) \geq{ }^{s} J_{F, a_{1}}^{\frac{\alpha}{k}}\left[\left(\tau-a_{1}\right)^{\sigma} h(\tau)\right]^{s} J_{F, a_{1}}^{\frac{\alpha}{k}}\left[h(\tau) \varphi^{\theta}(\tau)\right]$.

Proof. Since the function $\varphi$ is positive continuous and non-decreasing on $\left[a_{1}, a_{2}\right]$ then, for all $\theta>0, \sigma>0, u, v \in\left[a_{1}, \tau\right]$, with $v \leq u$, and $a_{1}<\tau \leq a_{2}$, we have

$$
\left(\left(u-a_{1}\right)^{\sigma}-\left(v-a_{1}\right)^{\sigma}\right)\left(\varphi^{\theta}(u)-\varphi^{\theta}(v)\right) \geq 0 .
$$


So, from (5) we get

$$
\left(u-a_{1}\right)^{\sigma} \varphi^{\theta}(u)+\left(v-a_{1}\right)^{\sigma} \varphi^{\theta}(v) \geq\left(u-a_{1}\right)^{\sigma} \varphi^{\theta}(v)+\left(v-a_{1}\right)^{\sigma} \varphi^{\theta}(u) .
$$

Multiplying both sides of 66 by $\frac{F(u, s) h(u)}{k \Gamma_{k}(\alpha)[\mathbb{F}(\tau, u)]^{1-\frac{\alpha}{k}}}$, then we integrate the resulting inequality with respect to $u$ over $\left(a_{1}, \tau\right)$, we get

$$
\begin{array}{r}
{ }^{s} J_{F, a_{1}}^{\frac{\alpha}{k}}\left[\left(\tau-a_{1}\right)^{\sigma} h(\tau) \varphi^{\theta}(\tau)\right]+\left(v-a_{1}\right)^{\sigma} \varphi^{\theta}(v)^{s} J_{F, a_{1}}^{\frac{\alpha}{k}} h(\tau) \geq \varphi^{\theta}(v)^{s} J_{F, a_{1}}^{\frac{\alpha}{k}}\left[\left(\tau-a_{1}\right)^{\sigma} h(\tau)\right] \\
+\left(v-a_{1}\right)^{\sigma} s J_{F, a_{1}}^{\frac{\alpha}{k}}\left[h(\tau) \varphi^{\theta}(\tau)\right] . \quad(7)
\end{array}
$$

Now, multiplying both sides of $\sqrt{7}$ by $\frac{F(v, s) h(v)}{k \Gamma_{k}(\alpha)[\mathbb{F}(\tau, v)]^{1-\frac{\alpha}{k}}}$, then we integrate the resulting inequality with respect to $v$ over $\left(a_{1}, \tau\right)$, it holds that

$$
\begin{aligned}
& { }^{s} J_{F, a_{1}}^{\frac{\alpha}{k}} h(\tau){ }^{s} J_{F, a_{1}}^{\frac{\alpha}{k}}\left[\left(\tau-a_{1}\right)^{\sigma} h(\tau) \varphi^{\theta}(\tau)\right]+{ }^{s} J_{F, a_{1}}^{\frac{\alpha}{k}}\left[\left(\tau-a_{1}\right)^{\sigma} h(\tau) \varphi^{\theta}(\tau)\right]{ }^{s} J_{F, a_{1}}^{\frac{\alpha}{k}} h(\tau) \geq \\
& { }^{s} J_{F, a_{1}}^{\frac{\alpha}{k}}\left[\left(\tau-a_{1}\right)^{\sigma} h(\tau)\right]^{s} J_{F, a_{1}}^{\frac{\alpha}{k}}\left[h(\tau) \varphi^{\theta}(\tau)\right]+{ }^{s} J_{F, a_{1}}^{\frac{\alpha}{k}}\left[h(\tau) \varphi^{\theta}(\tau)\right]^{s} J_{F, a_{1}}^{\frac{\alpha}{k}}\left[\left(\tau-a_{1}\right)^{\sigma} h(\tau)\right],
\end{aligned}
$$

which implies (4).

Remark 3. If we take the kernel $F(\tau, s)=\tau^{s}$, with $k>0$ and $s \in \mathbb{R}, s \neq-1$, the previous theorem becomes the Theorem 6 of [5].

In the following result, two functional parameters are used, extending the previous theorem.

Theorem 4. Let $\varphi$ be a positive non-decreasing continuous function on $\left[a_{1}, a_{2}\right]$ and let $h:\left[a_{1}, a_{2}\right] \rightarrow \mathbb{R}^{+}$be a positive continuous function. Then for $a_{1}<\tau \leq a_{2}$, $\theta>0, \sigma>0, \alpha>0, \beta>0$ and $s \neq-1$, we have

$$
\begin{gathered}
{ }^{s} J_{F, a_{1}}^{\frac{\alpha}{k}}\left[\left(\tau-a_{1}\right)^{\sigma} h(\tau) \varphi^{\theta}(\tau)\right]{ }^{s} J_{F, a_{1}}^{\frac{\beta}{k}} h(\tau)+{ }^{s} J_{F, a_{1}}^{\frac{\beta}{k}}\left[\left(\tau-a_{1}\right)^{\sigma} h(\tau) \varphi^{\theta}(\tau)\right]{ }^{s} J_{F, a_{1}}^{\frac{\alpha}{k}} h(\tau) \\
\geq{ }^{s} J_{F, a_{1}}^{\frac{\beta}{k}}\left[h(\tau) \varphi^{\theta}(\tau)\right]{ }^{s} J_{F, a_{1}}^{\frac{\alpha}{k}}\left[\left(\tau-a_{1}\right)^{\sigma} h(\tau)\right]+ \\
{ }^{s} J_{F, a_{1}}^{\frac{\beta}{k}}\left[\left(\tau-a_{1}\right)^{\sigma} h(\tau)\right]{ }^{s} J_{F, a_{1}}^{\frac{\alpha}{k}}\left[h(\tau) \varphi^{\theta}(\tau)\right] .
\end{gathered}
$$

Proof. Multiplying both sides of $\sqrt{7}$ by $\frac{F(v, s) h(v)}{k \Gamma_{k}(\beta)[\mathbb{F}(\tau, v)]^{1-\frac{\beta}{k}}}$, then we integrate the resulting inequality with respect to $v$ over $\left(a_{1}, \tau\right)$, we obtain (9).

Remark 5. If in Theorem 2 we take $\alpha=\beta$, we obtain Theorem 4

Remark 6. Under the same conditions as the previous Remark, this result covers Theorem 7 of [5].

The following result generalizes the Theorem 2, by including an appropriate $h$ function. 
Theorem 7. Let $\varphi$ and $\psi$ be two positive continuous function on $\left[a_{1}, a_{2}\right]$ such that $\varphi$ is non-decreasing, $\psi$ is non-increasing, and let $h:\left[a_{1}, a_{2}\right] \rightarrow \mathbb{R}^{+}$be a positive continuous function. Then for $a_{1}<\tau \leq a_{2}, \theta>0, \sigma>0, \alpha>0$ and $s \neq-1$, we have

$$
{ }^{s} J_{F, a_{1}}^{\frac{\alpha}{k}}\left[h(\tau) \psi^{\sigma}(\tau)\right]^{s} J_{F, a_{1}}^{\frac{\alpha}{k}}\left[h(\tau) \varphi^{\theta}(\tau)\right] \geq{ }^{s} J_{F, a_{1}}^{\frac{\alpha}{k}} h(\tau){ }^{s} J_{F, a_{1}}^{\frac{\alpha}{k}}\left[h(\tau) \varphi^{\theta}(\tau) \psi^{\sigma}(\tau)\right]
$$

Proof. Since the functions $\varphi$ and $\psi$ are positive continuous on $\left[a_{1}, a_{2}\right]$ with $\varphi$ is non-decreasing and $\psi$ is non-increasing, then for all $\theta>0, \sigma>0, u, v \in\left[a_{1}, \tau\right]$, with $v \leq u$, and $a_{1}<\tau \leq a_{2}$, we have

$$
\varphi^{\theta}(u) \psi^{\sigma}(v)+\varphi^{\theta}(v) \psi^{\sigma}(u) \geq \varphi^{\theta}(u) \psi^{\sigma}(u)+\varphi^{\theta}(v) \psi^{\sigma}(v) .
$$

Multiplying 11 by $\frac{F(u, s) h(u)}{k \Gamma_{k}(\alpha)[\mathbb{F}(\tau, u)]^{1-\frac{\alpha}{k}}}$, then we integrate the resulting inequality with respect to $u$ over $\left(a_{1}, \tau\right)$, it holds that

$$
\begin{aligned}
\psi^{\sigma}(v){ }^{s} J_{F, a_{1}}^{\frac{\alpha}{k}}\left[h(\tau) \varphi^{\theta}(\tau)\right]+\varphi^{\theta}(v){ }^{s} J_{F, a_{1}}^{\frac{\alpha}{k}}\left[h(\tau) \psi^{\sigma}(\tau)\right] & \geq{ }^{s} J_{F, a_{1}}^{\frac{\alpha}{k}}\left[h(\tau) \varphi^{\theta}(\tau) \psi^{\sigma}(\tau)\right] \\
& +\varphi^{\theta}(v) \psi^{\sigma}(v){ }^{s} J_{F, a_{1}}^{\frac{\alpha}{k}} h(\tau) .
\end{aligned}
$$

Now, we multiple 12 by $\frac{F(v, s) h(v)}{k \Gamma_{k}(\alpha)[\mathbb{F}(\tau, v)]^{1-\frac{\alpha}{k}}}$, then we integrate the resulting inequality with respect to $v$ over $\left(a_{1}, \tau\right)$, we get

$$
\begin{gathered}
{ }^{s} J_{F, a_{1}}^{\frac{\alpha}{k}}\left[h(\tau) \psi^{\sigma}(\tau)\right]{ }^{s} J_{F, a_{1}}^{\frac{\alpha}{k}}\left[h(\tau) \varphi^{\theta}(\tau)\right]+{ }^{s} J_{F, a_{1}}^{\frac{\alpha}{k}}\left[h(\tau) \varphi^{\theta}(\tau)\right]{ }^{s} J_{F, a_{1}}^{\frac{\alpha}{k}}\left[h(\tau) \psi^{\sigma}(\tau)\right] \geq \\
{ }^{s} J_{F, a_{1}}^{\frac{\alpha}{k}} h(\tau){ }^{s} J_{F, a_{1}}^{\frac{\alpha}{k}}\left[h(\tau) \varphi^{\theta}(\tau) \psi^{\sigma}(\tau)\right]+{ }^{s} J_{F, a_{1}}^{\frac{\alpha}{k}}\left[h(\tau) \varphi^{\theta}(\tau) \psi^{\sigma}(\tau)\right]{ }^{s} J_{F, a_{1}}^{\frac{\alpha}{k}} h(\tau),
\end{gathered}
$$

which implies 10.

Remark 8. As before, if we have $F(\tau, s)=\tau^{s}$, with $k>0$ and $s \in \mathbb{R}, s \neq-1$, this theorem reduces to Theorem 9 of [5].

Theorem 9. Let $\varphi$ and $\psi$ be two positive continuous function on $\left[a_{1}, a_{2}\right]$ such that $\varphi$ is non-decreasing, $\psi$ is non-increasing, and let $h:\left[a_{1}, a_{2}\right] \rightarrow \mathbb{R}^{+}$be a positive continuous function. Then for $a_{1}<\tau \leq a_{2}, \theta>0, \sigma>0, \beta>0, \alpha>0$ and $s \neq-1$, we have

$$
\begin{gathered}
s J_{F, a_{1}}^{\frac{\beta}{k}}\left[h(\tau) \psi^{\sigma}(\tau)\right]{ }^{s} J_{F, a_{1}}^{\frac{\alpha}{k}}\left[h(\tau) \varphi^{\theta}(\tau)\right]+{ }^{s} J_{F, a_{1}}^{\frac{\beta}{k}}\left[h(\tau) \varphi^{\theta}(\tau)\right]{ }^{s} J_{F, a_{1}}^{\frac{\alpha}{k}}\left[h(\tau) \psi^{\sigma}(\tau)\right] \geq \\
s J_{F, a_{1}}^{\frac{\beta}{k}} h(\tau){ }^{s} J_{F, a_{1}}^{\frac{\alpha}{k}}\left[h(\tau) \varphi^{\theta}(\tau) \psi^{\sigma}(\tau)\right]+{ }^{s} J_{F, a_{1}}^{\frac{\beta}{k}}\left[h(\tau) \varphi^{\theta}(\tau) \psi^{\sigma}(\tau)\right]^{s} J_{F, a_{1}}^{\frac{\alpha}{k}} h(\tau) .
\end{gathered}
$$

Proof. If we multiple 12 by $\frac{F(v, s) h(v)}{k \Gamma_{k}(\beta)[\mathbb{F}(\tau, v)]^{1-\frac{\beta}{k}}}$, then we integrate the resulting inequality with respect to $v$ over $\left(a_{1}, \tau\right)$, we obtain 13 . 
Remark 10. If in Theorem 9 we put $\alpha=\beta$ we obtain Theorem 7 ,

Remark 11. Theorem 10 of [5] is obtained from Theorema 9, considering, as before, the kernel $F(\tau, s)=\tau^{s}$, with $k>0$ and $s \in \mathbb{R}, s \neq-1$.

Theorem 12. Let $\varphi$ be a positive decreasing continuous function on $\left[a_{1}, a_{2}\right]$ and let $h:\left[a_{1}, a_{2}\right] \rightarrow \mathbb{R}^{+}$be a positive continuous function. Then for $a_{1}<\tau \leq a_{2}$, $\delta \geq \theta>0, \sigma>0, \alpha>0$ and $s \neq-1$, we have

$$
\begin{aligned}
& { }^{s} J_{F, a_{1}}^{\frac{\alpha}{k}}\left[\left(\tau-a_{1}\right)^{\sigma} h(\tau) \varphi^{\theta}(\tau)\right]{ }^{s} J_{F, a_{1}}^{\frac{\alpha}{k}}\left[h(\tau) \varphi^{\delta}(\tau)\right] \geq \\
& { }^{s} J_{F, a_{1}}^{\frac{\alpha}{k}}\left[\left(\tau-a_{1}\right)^{\sigma} h(\tau) \varphi^{\delta}(\tau){ }^{s} J_{F, a_{1}}^{\frac{\alpha}{k}}\left[h(\tau) \varphi^{\theta}(\tau)\right] .\right.
\end{aligned}
$$

Proof. Since the function $\varphi$ is positive continuous and decreasing on $\left[a_{1}, a_{2}\right]$ then, for all $\delta-\theta \geq 0, \sigma>0, u, v \in\left[a_{1}, \tau\right]$, with $v \leq u$, and $a_{1}<\tau \leq a_{2}$, we have

$$
\left(u-a_{1}\right)^{\sigma} \varphi^{\delta-\theta}(v)+\left(v-a_{1}\right)^{\sigma} \varphi^{\delta-\theta}(u) \geq\left(u-a_{1}\right)^{\sigma} \varphi^{\delta-\theta}(u)+\left(v-a_{1}\right)^{\sigma} \varphi^{\delta-\theta}(v) .
$$

Multiplying both sides of 15 by $\frac{F(u, s) h(u) \varphi^{\theta}(u)}{k \Gamma_{k}(\alpha)[\mathbb{F}(\tau, u)]^{1-\frac{\alpha}{k}}}$, then we integrate the resulting inequality with respect to $u$ over $\left(a_{1}, \tau\right)$, we get

$$
\begin{aligned}
& \varphi^{\delta-\theta}(v){ }^{s} J_{F, a_{1}}^{\frac{\alpha}{k}}\left[\left(\tau-a_{1}\right)^{\sigma} h(\tau) \varphi^{\theta}(\tau)\right]+\left(v-a_{1}\right)^{\sigma s} J_{F, a_{1}}^{\frac{\alpha}{k}}\left[h(\tau) \varphi^{\delta}(\tau)\right] \geq \\
& { }^{s} J_{F, a_{1}}^{\frac{\alpha}{k}}\left[\left(\tau-a_{1}\right)^{\sigma} h(\tau) \varphi^{\delta}(\tau)\right]+\left(v-a_{1}\right)^{\sigma} \varphi^{\delta-\theta}(v)^{s} J_{F, a_{1}}^{\frac{\alpha}{k}}\left[h(\tau) \varphi^{\theta}(\tau)\right] .
\end{aligned}
$$

Now, multiplying both sides of 16 by $\frac{F(v, s) h(v) \varphi^{\theta}(v)}{k \Gamma_{k}(\alpha)[\mathbb{F}(\tau, v)]^{1-\frac{\alpha}{k}}}$, then we integrate the resulting inequality with respect to $v$ over $\left(a_{1}, \tau\right)$, it holds that

$$
\begin{array}{r}
{ }^{s} J_{F, a_{1}}^{\frac{\alpha}{k}}\left[h(\tau) \varphi^{\delta}(\tau)\right]{ }^{s} J_{F, a_{1}}^{\frac{\alpha}{k}}\left[\left(\tau-a_{1}\right)^{\sigma} h(\tau) \varphi^{\theta}(\tau)\right]+ \\
{ }^{s} J_{F, a_{1}}^{\frac{\alpha}{k}}\left[\left(\tau-a_{1}\right)^{\sigma} h(\tau) \varphi^{\theta}(\tau)\right]{ }^{s} J_{F, a_{1}}^{\frac{\alpha}{k}}\left[h(\tau) \varphi^{\delta}(\tau)\right] \geq \\
{ }^{s} J_{F, a_{1}}^{\frac{\alpha}{k}}\left[h(\tau) \varphi^{\theta}(\tau)\right]{ }^{s} J_{F, a_{1}}^{\frac{\alpha}{k}}\left[\left(\tau-a_{1}\right)^{\sigma} h(\tau) \varphi^{\delta}(\tau)\right]+ \\
{ }^{s} J_{F, a_{1}}^{\frac{\alpha}{k}}\left[\left(\tau-a_{1}\right)^{\sigma} h(\tau) \varphi^{\delta}(\tau)\right]{ }^{s} J_{F, a_{1}}^{\frac{\alpha}{k}}\left[h(\tau) \varphi^{\theta}(\tau)\right],
\end{array}
$$

which implies (14).

Remark 13. This Theorem contains as a particular case Theorem 12 of [5], under the same conditions as the previous Remarks.

The latest results of our work is the extension of the previous Theorems considering the product of a family of appropriate functions.

Theorem 14. Let $\varphi_{j}, j=1,2, \ldots, n$ are $n$ positive continuous and decreasing functions on $\left[a_{1}, a_{2}\right]$ and let $h:\left[a_{1}, a_{2}\right] \rightarrow \mathbb{R}^{+}$be a positive continuous function. Then for $a_{1}<\tau \leq a_{2}, \alpha>0, s \neq-1, \sigma>0$ and $\delta \geq \theta_{r}>0$ with $r \in\{1,2, \ldots, n\}$, we have 


$$
\begin{gathered}
s J_{F, a_{1}}^{\frac{\alpha}{k}}\left[h(\tau) \varphi_{r}^{\delta}(\tau) \prod_{j \neq r}^{n} \varphi_{j}^{\theta_{j}}(\tau)\right] s J_{F, a_{1}}^{\frac{\alpha}{k}}\left[\left(\tau-a_{1}\right)^{\sigma} h(\tau) \prod_{j=1}^{n} \varphi_{j}^{\theta_{j}}(\tau)\right] \geq \\
s J_{F, a_{1}}^{\frac{\alpha}{k}}\left[h(\tau) \prod_{j=1}^{n} \varphi_{j}^{\theta_{j}}(\tau)\right] s J_{F, a_{1}}^{\frac{\alpha}{k}}\left[\left(\tau-a_{1}\right)^{\sigma} h(\tau) \varphi_{r}^{\delta}(\tau) \prod_{j \neq r}^{n} \varphi_{j}^{\theta_{j}}(\tau)\right] .
\end{gathered}
$$

Proof. Since the function $\varphi_{r}$ is positive continuous and decreasing on $\left[a_{1}, a_{2}\right]$ then, for all $\sigma>0, a_{1}<\tau \leq a_{2}, u, v \in\left[a_{1}, \tau\right]$, with $v \leq u$, and $\delta \geq \theta_{r}>0$, for any fixed $r \in\{1,2, \ldots, n\}$, we get

$$
\left(\left(u-a_{1}\right)^{\sigma}-\left(v-a_{1}\right)^{\sigma}\right)\left(\varphi_{r}^{\delta-\theta_{r}}(v)-\varphi_{r}^{\delta-\theta_{r}}(u)\right) \geq 0 .
$$

So, from 19), we deduce that

$$
\left(u-a_{1}\right)^{\sigma} \varphi_{r}^{\delta-\theta_{r}}(v)+\left(v-a_{1}\right)^{\sigma} \varphi_{r}^{\delta-\theta_{r}}(u) \geq\left(u-a_{1}\right)^{\sigma} \varphi_{r}^{\delta-\theta_{r}}(u)+\left(v-a_{1}\right)^{\sigma} \varphi_{r}^{\delta-\theta_{r}}(v) .
$$

Multiplying both sides of 20 by $\frac{F(u, s) h(u) \prod_{j=1}^{n} \varphi_{j}^{\theta_{j}}(u)}{k \Gamma_{k}(\alpha)[\mathbb{F}(\tau, u)]^{1-\frac{\alpha}{k}}}$, then we integrate the resulting inequality with respect to $u$ over $\left(a_{1}, \tau\right)$, we get

$$
\begin{aligned}
& \varphi_{r}^{\delta-\theta_{r}}(v){ }^{s} J_{F, a_{1}}^{\frac{\alpha}{k}}\left[\left(\tau-a_{1}\right)^{\sigma} h(\tau) \prod_{j=1}^{n} \varphi_{j}^{\theta_{j}}(\tau)\right]+ \\
& \left(v-a_{1}\right)^{\sigma s} J_{F, a_{1}}^{\frac{\alpha}{k}}\left[h(\tau) \varphi_{r}^{\delta}(\tau) \prod_{j \neq r}^{n} \varphi_{j}^{\theta_{j}}(\tau)\right] \geq \\
& { }^{s} J_{F, a_{1}}^{\frac{\alpha}{k}}\left[\left(\tau-a_{1}\right)^{\sigma} h(\tau) \varphi_{r}^{\delta}(\tau) \prod_{j \neq r}^{n} \varphi_{j}^{\theta_{j}}(\tau)\right]+ \\
& \left(v-a_{1}\right)^{\sigma} \varphi_{r}^{\delta-\theta_{r}}(v){ }^{s} J_{F, a_{1}}^{\frac{\alpha}{k}}\left[h(\tau) \prod_{j=1}^{n} \varphi_{j}^{\theta_{j}}(\tau)\right] .
\end{aligned}
$$

Lastly, multiplying both sides of 21 by $\frac{F(v, s) h(v) \prod_{j=1}^{n} \varphi_{j}{ }^{\theta_{j}}(v)}{k \Gamma_{k}(\alpha)[\mathbb{F}(\tau, v)]^{1-\frac{\alpha}{k}}}$, then we integrate the resulting inequality with respect to $v$ over $\left(a_{1}, \tau\right)$, which concludes the inequality (18).

Theorem 15. Let $\varphi_{j}, j=1,2, \ldots, n$ are $n$ positive continuous and decreasing functions on $\left[a_{1}, a_{2}\right]$ and let $h:\left[a_{1}, a_{2}\right] \rightarrow \mathbb{R}^{+}$be a positive continuous function. Then for $a_{1}<\tau \leq a_{2}, \alpha>0, \beta>0, s \neq-1, \sigma>0$ and $\delta \geq \theta_{r}>0$ with $r \in\{1,2, \ldots, n\}$, we have

$$
{ }^{s} J_{F, a_{1}}^{\frac{\alpha}{k}}\left[\left(\tau-a_{1}\right)^{\sigma} h(\tau) \prod_{j=1}^{n} \varphi_{j}^{\theta_{j}}(\tau)\right]{ }^{s} J_{F, a_{1}}^{\frac{\beta}{k}}\left[h(\tau) \varphi_{r}{ }^{\delta}(\tau) \prod_{j \neq r}^{n} \varphi_{j}^{\theta_{j}}(\tau)\right]
$$




$$
\begin{gathered}
+{ }^{s} J_{F, a_{1}}^{\frac{\alpha}{k}}\left[h(\tau) \varphi_{r}{ }^{\delta}(\tau) \prod_{j \neq r}^{n} \varphi_{j}^{\theta_{j}}(\tau)\right] s J_{F, a_{1}}^{\frac{\beta}{k}}\left[\left(\tau-a_{1}\right)^{\sigma} h(\tau) \prod_{j=1}^{n} \varphi_{j}^{\theta_{j}}(\tau)\right] \geq \\
s^{s} J_{F, a_{1}}^{\frac{\alpha}{k}}\left[\left(\tau-a_{1}\right)^{\sigma} h(\tau) \varphi_{r}{ }^{\delta}(\tau) \prod_{j \neq r}^{n} \varphi_{j}^{\theta_{j}}(\tau)\right] s J_{F, a_{1}}^{\frac{\beta}{k}}\left[h(\tau) \prod_{j=1}^{n} \varphi_{j}^{\theta_{j}}(\tau)\right] \\
+{ }^{s} J_{F, a_{1}}^{\frac{\alpha}{k}}\left[h(\tau) \prod_{j=1}^{n} \varphi_{j}{ }^{\theta_{j}}(\tau)\right]{ }^{s} J_{F, a_{1}}^{\frac{\beta}{k}}\left[\left(\tau-a_{1}\right)^{\sigma} h(\tau) \varphi_{r}{ }^{\delta}(\tau) \prod_{j \neq r}^{n} \varphi_{j}^{\theta_{j}}(\tau)\right] .
\end{gathered}
$$

Proof. If we multiple both sides of 21 by $\frac{F(v, s) h(v) \prod_{j=1}^{n} \varphi_{j}^{\theta_{j}}(v)}{k \Gamma_{k}(\alpha)[\mathbb{F}(\tau, v)]^{1-\frac{\beta}{k}}}$, then we integrate the resulting inequality with respect to $v$ over $\left(a_{1}, \tau\right)$, with which we deduce the inequality 22 .

Remark 16. If in Theorem 15 we put $\alpha=\beta$ we obtain Theorem 14 .

Theorem 17. Let $\varphi_{j}, j=1,2, \ldots, n$ and $\psi$ are positive continuous functions on $\left[a_{1}, a_{2}\right]$, such that $\psi$ is increasing and $\varphi_{j}, j=1,2, \ldots, n$ are decreasing on $\left[a_{1}, a_{2}\right]$ and let $h:\left[a_{1}, a_{2}\right] \rightarrow \mathbb{R}^{+}$be a positive continuous function. Then for $a_{1}<\tau \leq a_{2}$, $\alpha>0, s \neq-1, \sigma>0$ and $\delta \geq \theta_{r}>0$ with $r \in\{1,2, \ldots, n\}$, we have

$$
\begin{aligned}
s J_{F, a_{1}}^{\frac{\alpha}{k}} & {\left[h(\tau) \varphi_{r}{ }^{\delta}(\tau) \prod_{j \neq r}^{n} \varphi_{j}^{\theta_{j}}(\tau)\right]{ }^{s} J_{F, a_{1}}^{\frac{\alpha}{k}}\left[\psi^{\sigma}(\tau) h(\tau) \prod_{j=1}^{n} \varphi_{j}^{\theta_{j}}(\tau)\right] \geq } \\
& s J_{F, a_{1}}^{\frac{\alpha}{k}}\left[h(\tau) \prod_{j=1}^{n} \varphi_{j}^{\theta_{j}}(\tau)\right]{ }^{s} J_{F, a_{1}}^{\frac{\alpha}{k}}\left[\psi^{\sigma}(\tau) h(\tau) \varphi_{r}{ }^{\delta}(\tau) \prod_{j \neq r}^{n} \varphi_{j}^{\theta_{j}}(\tau)\right] .
\end{aligned}
$$

Proof. Since the functions $\varphi_{r}$ and $\psi$ are positive continuous on $\left[a_{1}, a_{2}\right]$ with $\varphi_{r}$ decreasing and $\psi$ increasing; then, for all $\sigma>0, a_{1}<\tau \leq a_{2}, u, v \in\left[a_{1}, \tau\right]$, with $v \leq u$, and $\delta \geq \theta_{r}>0$, for any fixed $r \in\{1,2, \ldots, n\}$, we get

$$
\psi^{\sigma}(u) \varphi_{r}^{\delta-\theta_{r}}(v)+\psi^{\sigma}(v) \varphi_{r}^{\delta-\theta_{r}}(u) \geq \psi^{\sigma}(u) \varphi_{r}^{\delta-\theta_{r}}(u)+\psi^{\sigma}(v) \varphi_{r}^{\delta-\theta_{r}}(v) .
$$

Multiplying both sides of 24 by $\frac{F(u, s) h(u) \prod_{j=1}^{n} \varphi_{j}{ }^{\theta_{j}}(u)}{k \Gamma_{k}(\alpha)[\mathbb{F}(\tau, u)]^{1-\frac{\alpha}{k}}}$, then we integrate the resulting inequality with respect to $u$ over $\left(a_{1}, \tau\right)$, we deduce

$$
\begin{gathered}
\varphi_{r}^{\delta-\theta_{r}}(v)^{s} J_{F, a_{1}}^{\frac{\alpha}{k}}\left[\psi^{\sigma}(\tau) h(\tau) \prod_{j=1}^{n} \varphi_{j}^{\theta_{j}}(\tau)\right]+\psi^{\sigma}(v)^{s} J_{F, a_{1}}^{\frac{\alpha}{k}}\left[h(\tau) \varphi_{r}^{\delta}(\tau) \prod_{j \neq r}^{n} \varphi_{j}^{\theta_{j}}(\tau)\right] \geq \\
{ }^{s} J_{F, a_{1}}^{\frac{\alpha}{k}}\left[\psi^{\sigma}(\tau) h(\tau) \varphi_{r}^{\delta}(\tau) \prod_{j \neq r}^{n} \varphi_{j}^{\theta_{j}}(\tau)\right]+\psi^{\sigma}(v) \varphi_{r}^{\delta-\theta_{r}}(v)^{s} J_{F, a_{1}}^{\frac{\alpha}{k}}\left[h(\tau) \prod_{j=1}^{n} \varphi_{j}^{\theta_{j}}(\tau)\right] .
\end{gathered}
$$


Lastly, multiplying both sides of 25 by $\frac{F(v, s) h(v) \prod_{j=1}^{n} \varphi_{j}^{\theta_{j}}(v)}{k \Gamma_{k}(\alpha)[\mathbb{F}(\tau, v)]^{1-\frac{\alpha}{k}}}$, then we integrate the resulting inequality with respect to $v$ over $\left(a_{1}, \tau\right)$, which concludes the inequality 23 .

Theorem 18. Let $\varphi_{j}, j=1,2, \ldots, n$ and $\psi$ are positive continuous functions on $\left[a_{1}, a_{2}\right]$, such that $\psi$ is increasing and $\varphi_{j}, j=1,2, \ldots, n$ are decreasing on $\left[a_{1}, a_{2}\right]$ and let $h:\left[a_{1}, a_{2}\right] \rightarrow \mathbb{R}^{+}$be a positive continuous function. Then for $a_{1}<\tau \leq a_{2}$, $\beta>0, \alpha>0, s \neq-1, \sigma>0$ and $\delta \geq \theta_{r}>0$ with $r \in\{1,2, \ldots, n\}$, we have

$$
\begin{gathered}
s J_{F, a_{1}}^{\frac{\alpha}{k}}\left[h(\tau) \varphi_{r}^{\delta}(\tau) \prod_{j \neq r}^{n} \varphi_{j}^{\theta_{j}}(\tau)\right] s J_{F, a_{1}}^{\frac{\beta}{k}}\left[\psi^{\sigma}(\tau) h(\tau) \prod_{j=1}^{n} \varphi_{j}^{\theta_{j}}(\tau)\right] \\
+{ }^{s} J_{F, a_{1}}^{\frac{\beta}{k}}\left[h(\tau) \varphi_{r}^{\delta}(\tau) \prod_{j \neq r}^{n} \varphi_{j}^{\theta_{j}}(\tau)\right]{ }^{s} J_{F, a_{1}}^{\frac{\alpha}{k}}\left[\psi^{\sigma}(\tau) h(\tau) \prod_{j=1}^{n} \varphi_{j}^{\theta_{j}}(\tau)\right] \geq \\
{ }^{s} J_{F, a_{1}}^{\frac{\alpha}{k}}\left[h(\tau) \prod_{j=1}^{n} \varphi_{j}^{\theta_{j}}(\tau)\right]{ }^{s} J_{F, a_{1}}^{\frac{\beta}{k}}\left[\psi^{\sigma}(\tau) h(\tau) \varphi_{r}{ }^{\delta}(\tau) \prod_{j \neq r}^{n} \varphi_{j}^{\theta_{j}}(\tau)\right] \\
+{ }^{s} J_{F, a_{1}}^{\frac{\beta}{k}}\left[h(\tau) \prod_{j=1}^{n} \varphi_{j}^{\theta_{j}}(\tau)\right] s^{s} J_{F, a_{1}}^{\frac{\alpha}{k}}\left[\psi^{\sigma}(\tau) h(\tau) \varphi_{r}{ }^{\delta}(\tau) \prod_{j \neq r}^{n} \varphi_{j}^{\theta_{j}}(\tau)\right] .
\end{gathered}
$$

Proof. If we multiple both sides of 25 by $\frac{F(v, s) h(v) \prod_{j=1}^{n} \varphi_{j}{ }^{\theta_{j}}(v)}{k \Gamma_{k}(\alpha)[\mathbb{F}(\tau, v)]^{1-\frac{\beta}{k}}}$, then we integrate the resulting inequality with respect to $v$ over $\left(a_{1}, \tau\right)$, with which we deduce the inequality (26).

Remark 19. If in Theorem 18 we put $\alpha=\beta$ we obtain Theorem 17 .

Remark 20. Theorems 14, 15, 17 and 18, are generalizations of Theorems 18, 19, 21 and 22 of [5], respectively.

\section{CONCLUSIONS}

In this work we have obtained various inequalities of the Hermite-Hadamard type, in the case of different notions of convexity, and using a generalized fractional operator, which allows obtaining as particular cases, several of those reported in the literature.

We want to point out, in addition to the observations made throughout the work, that with different choices of the $F$ kernel we can obtain, as particular cases, several well-known integral operators. So, for example, if

A) The classic Riemann integral is obtained with $F(t, \alpha)=t^{\alpha-1}, \alpha=1$ and $\beta=k$ (with notation changed). 
B) If $F(t, \alpha)=t^{\alpha-1}$ and $\beta=k$ we obtain the fractional Riemann-Liouville integral.

C) Considering $F(t, \alpha)=t^{s}$ with $s=1$, we can write the right sided operator as follows $\left(\begin{array}{cc}\frac{\beta}{k} & J_{F, a+}^{\alpha} f\end{array}\right)(x)=\frac{1}{k \Gamma_{k}(\beta)} \int_{a}^{x} \frac{f(t) d t}{(x-t)^{1-\frac{\beta}{k}}}$ and similarly the left sided integral. The k-Riemann-Liouville fractional integral of Mubeen and Habibullah (see $[7])$.

D) Katugampola fractional integral of 6 is obtained, taking $F(t, \alpha)=t^{-\alpha}$ (the notation is changed).

E) If we put $F=t^{-s}$ with $s=1$, then we get the right sided Hadamard fractional integral of [4].

F) An integral operator with non-singular kernel can also be obtained from our Definition 1. Thus, considering $F(t, \alpha)=\exp \left[-\frac{1-\alpha}{\alpha} t\right]$, if $\alpha=1$ we have that $F=1$. In this case $F\left(\mathbb{F}_{+}(x, t), \beta\right)=\exp \left[\frac{1-\beta}{\beta}(x-t)\right]$, a slight modification of the operator defined by Kirane and Toberek in [1].

From all of the above, we can conclude that many of the integral inequalities obtained in the framework of these integral operators, can be obtained as particular cases from those obtained in this work.

Authors Contribution Statement The authors contributed equally to this work. All authors of the submitted research paper have directly participated in the planning, execution, or analysis of study.

Declaration of Competing Interest The authors declare that there is no conflict of interest regarding the publication of this article. Besides, the contents of the manuscript have not been submitted, copyrighted or published elsewhere and the visual-graphical materials such as photograph, drawing, picture, and document within the article do not have any copyright issue. Finally, all authors of the paper have read and approved the final version submitted.

\section{REFERENCES}

[1] Ahmad, B., Alsaedi, A., Kirane, M., Toberek, B. T., Hermite-Hadamard, Hermite-HadamarFejer, Dragomir-Agarwal and pachpatte tyoe inequalities for convex functions via new fractional integrals, ArXiv: 1701.00092

[2] Díaz, R., Pariguan, E., On hypergeometric functions and Pochhammer k-symbol, Divulg. Mat., 15(2) (2007), 179-192.

[3] Galeano, J., Nápoles, J., Pérez, E., On a general formulation of the fractional operator Riemann-Liouville and related inequalities, submitted.

[4] Hadamard, J., Étude sur les propriétés des fonctions entiéres et en particulier d'une fonction considerée par Riemann, J. Math. Pures Appl., 58 (1893), 171-216.

[5] Houas, M., Dahmani, Z., Sarikaya, M. Z., Some integral inequalities for (k,s) - RiemannLiouville fractional operators, Journal of Interdisciplinary Mathematics, 23(8) (2020), 14871495, https://doi.org/10.1080/09720502.2018.1478768. 
[6] Katugampola, U. N., New Approach Generalized Fractional Integral, Applied Math and Comp., 218 (2011), 860-865, https://doi.org/10.1016/j.amc.2011.03.062.

[7] Mubeen, S., Habibullah, G. M., K-fractional integrals and applications, Int. J. Contem. Math. Sci., 7(2) (2012), 89-94.

[8] Qi, F., Guo, B. N., Integral representations and complete monotonicity of remainders of the Binet and Stirling formulas for the gamma function, Rev. R. Acad. Cienc. Exactas FÃ-s. Nat., Ser. A Mat., 111(2) (2017), 425-434, https://doi.org/10.1007/s13398-016-0302-6.

[9] Rainville, E. D., Special Functions, Macmillan Co., New York, 1960.

[10] Yang, Z. H., Tian, J. F., Monotonicity and inequalities for the gamma function, J. Inequal. Appl., 317 (2017), https://doi.org/10.1186/s13660-017-1591-9.

[11] Yang, Z. H., Tian, J. F., Monotonicity and sharp inequalities related to gamma function, J. Math. Inequal., 12(1) (2018), 1-22, https://doi.org/10.7153/jmi-2018-12-01. 